\section{Kein Nutzen von Cranberrys bei Harnwegsinfekten}

Ein weiteres Mal nach 1998, 2004 und 2008 haben sich Forscher in einem Cochrane-Review der großfüchtigen Moosbeere gewidmet. Besser bekannt ist das Obst unter seiner englischen Bezeichnung Cranberry. Den Beeren respektive ihrem Saft wird nachgesagt, vorbeugend gegen Harnwegsinfekte zu wirken - offenbar größtenteils zu Unrecht.

$\mathrm{V}$ or vier Jahren hatte es noch nach einem geringen Nutzen von Cranberry-Saft zumindest bei Frauen mit rezidivierenden Harnwegsinfekten (HWI) ausgesehen. Seither sind Ergebnisse von 14 weiteren Studien mit dem Beerensaft veröffentlicht worden und in das neueste Cochrane-Review eingeflossen. Das Resümee fällt nun noch pessimistischer aus als im Update davor.

In keiner der untersuchten Gruppen konnte ein überzeugender Nutzen im Vergleich zu Placebo, Antibiotika, Laktobazillen oder keiner Kontrolltherapie nachgewiesen werden. $\mathrm{Zu}$ den insgesamt 4.473 Probanden in 24 Studien gehörten neben den erwähnten Frauen mit wiederkehrenden HWI weitere Risikogruppen: ältere Frauen und Männer, katheterisierte Patienten, Schwangere, Patienten mit Anomalien des Harntrakts und Kinder, die einen ersten oder rezidivierenden Infekt erlitten hatten. Neben Saft waren auch andere Produkte wie Tabletten und Kapseln getestet worden. Die Einnahmedauer betrug jeweils mindestens einen Monat.

Allenfalls bei Frauen mit rezidivierenden HWI ließ sich ein Trend erkennen, der einen Vorteil der Cranberry-Zubereitungen nahelegt. Das relative Risiko sank hier im Mittel um 26\%, das Konfidenzintervall reichte indes von einer Reduktion um $58 \%$ bis zu einer Steigerung von $31 \%$; damit war das Ergebnis nicht signifikant. Bemerkenswert war unter anderem die Quote der Therapieabbrüche, die in manchen Studien $55 \%$ erreichte. Das betraf besonders den Saft der Beeren, von dem teilweise ein Liter pro Tag zu leeren war. Die Cochrane-Autoren vermuten, dass sich mit Kapseln oder Tabletten die Compliance verbessern könnte. Allerdings lägen für diese Formen der Zubereitung aber nur wenige Studiendaten zur Wirkstoffmenge vor. Solche Cranberry-Produkte könnten daher nicht für die HWIPrävention empfohlen werden.

Fazit: Das britische Cochrane-Zentrum sieht keinen Nutzen von Cranberrys in der HWI-Prävention. Weitere Studien zu dem Thema sollten nicht mehr unternommen werden, meinen die Autoren, es sei denn, es sprächen starke Gründe dafür. Allenfalls Kapseln und Tabletten seien lohnende Studienobjekte, aber auch sie nur bei Frauen mit rezidivierenden HWI. Die Präparate müssten zudem mindestens eine tägliche Zufuhr von $36 \mathrm{mg}$ an Proanthocyanidinen gewährleisten.

Dr. Robert Bublak

Jepson RG et al. Cranberries for preventing urinary tract infections. Cochrane Database Syst Rev 2012; 10: CD001321

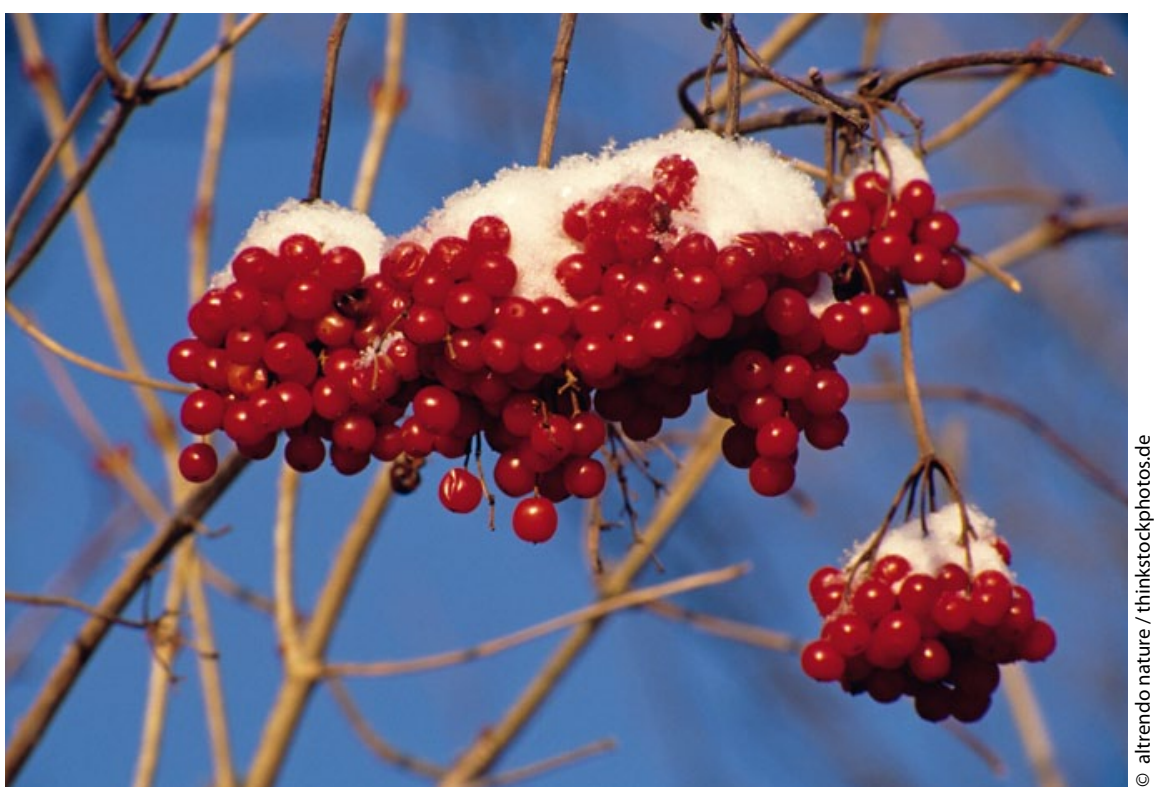

Cranberrys: laut Cochrane-Analyse zur HWI-Prävention eher ungeeignet 